\title{
Fifty Years of Seismic Network Performance in Greece (1964-2013): Spatiotemporal Evolution of the Completeness Magnitude
}

\section{by Arnaud Mignan and Gerasimos Chouliaras}

\section{INTRODUCTION}

The National Observatory of Athens (NOA) produces the NOA earthquake catalog since 1964 . For its 50 year anniversary, we describe the evolution of the Greek seismic network by examining its performance in terms of completeness magnitude $M_{\mathrm{c}}$.

Over its 50 years of existence, the earthquake catalog of Greece has improved on the basis of several network upgrades. The mid-1960s marked the start of the modern Greek seismic network coordinated by the NOA. Since then, the earthquake catalog of NOA has been published with no interruption. Three main upgrades of the network are notable. (1) The passage from analog-to-digital instrumentation and processing took place in 1995. (2) The development of the Hellenic Unified Seismological Network (HUSN) took place gradually from the end of 2007 to 2011, which combined the NOA network to three university networks (Athens, Patras, and Thessaloniki). In addition, (3) the upgrade of the magnitude determination software happened in early 2011. More information about the history and characteristics of the Greek seismic network can be found in the literature (Båth, 1983; Chouliaras and Stavrakakis, 1997; Papanastassiou et al., 2001; Papanastassiou, 2010; Roumelioti et al., 2010; D'Alessandro et al., 2011; Deshcherevskii and Sidorin, 2012; Chouliaras et al., 2013).

The goal of the present study is to provide the first comprehensive spatiotemporal analysis of the Greek seismic network performance in terms of completeness magnitude $M_{\mathrm{c}}$, computed using the recently proposed Bayesian magnitude of completeness (BMC) method (Mignan et al., 2011). We additionally make an in-depth analysis of the frequency-magnitude distribution (FMD) to validate the BMC results and to provide additional recommendations for the computation of $M_{\mathrm{c}}$.

\section{DATA SELECTION}

We used the NOA earthquake catalog, available at http://www .gein.noa.gr/en/seismicity/earthquake-catalogs (last accessed October 2013), and defined the study area $\left(19^{\circ} \mathrm{E} ; 29^{\circ} \mathrm{E}\right.$; $\left.34^{\circ} \mathrm{N} ; 42^{\circ} \mathrm{N}\right)$. We considered the seismic network of NOA (HL for Hellenic) for the period 1964-present with the station coordinates and start dates obtained from http://bbnet.gein. noa.gr/HL/real-time-plotting/noa-stations-list/hl-network-and- collaborative-stations-information (last accessed October 2013). For the remaining part of the HUSN, that is, Universities of Athens (HA), Patras (HP), and Thessaloniki (HT), we used the station coordinates available at http://bbnet.gein.noa.gr/HL/ real-time-plotting/onlinestations (last accessed October 2013). We obtained the starting date of all HA, HP, and HT stations in the HUSN from the NOA data server administration logs. In addition to the HUSN stations, we also considered five seismic stations located around Athens and operated between April 2004 and mid-2008 by the Greek Civil Protection. These stations were included in the daily analysis and bulletin production, and their dates of operation were obtained from the NOA monthly bulletins.

Figure 1 shows the evolution of the number of seismic stations, of the annual rate of earthquakes, and of the magnitude of completeness $M_{\mathrm{c}}$ over the period 1964-2013. $M_{\mathrm{c}}$ was computed using the median-based analysis of the segment slope (MBASS) method introduced by Amorèse (2007). We used a moving-window method with 1 year and 1 month windows. The mean and standard deviation of $M_{\mathrm{c}}$ were computed from 200 bootstrap samples. Use of the term "proxy to $M_{\mathrm{c}}$ " in Figures $1 \mathrm{c}$ and $1 \mathrm{~d}$ is explained later on in this paper. Based on the different metrics shown in Figure 1, we defined four periods [1964; 1995), [1995; 2008), [2008; 2011), and [2011; 2013], hereafter referred to as periods I, II, III, and IV, respectively. These periods are found in agreement with the main changes that occurred in the Greek seismic network over time.

\section{BAYESIAN MAGNITUDE OF COMPLETENESS (BMC)}

\footnotetext{
Method

We mapped the completeness magnitude $M_{\mathrm{c}}$ of the NOA catalog using the BMC method introduced by Mignan et al. (2011). This approach uses the robustness of Bayes theorem by combining local $M_{\mathrm{c}}$ observations with prior information based on the density of seismic stations. In contrast with other methods (see review by Mignan and Woessner, 2012), BMC provides a complete spatial coverage of $M_{\mathrm{c}}$ while avoiding oversmoothing. The method has already been successfully applied to Taiwan (Mignan et al., 2011), Mainland China (Mignan et al., 2013), the Lesser Antilles arc (Vorobieva et al., 2013), and Switzerland (Kraft et al., 2013).
} 
(a)

HUSN

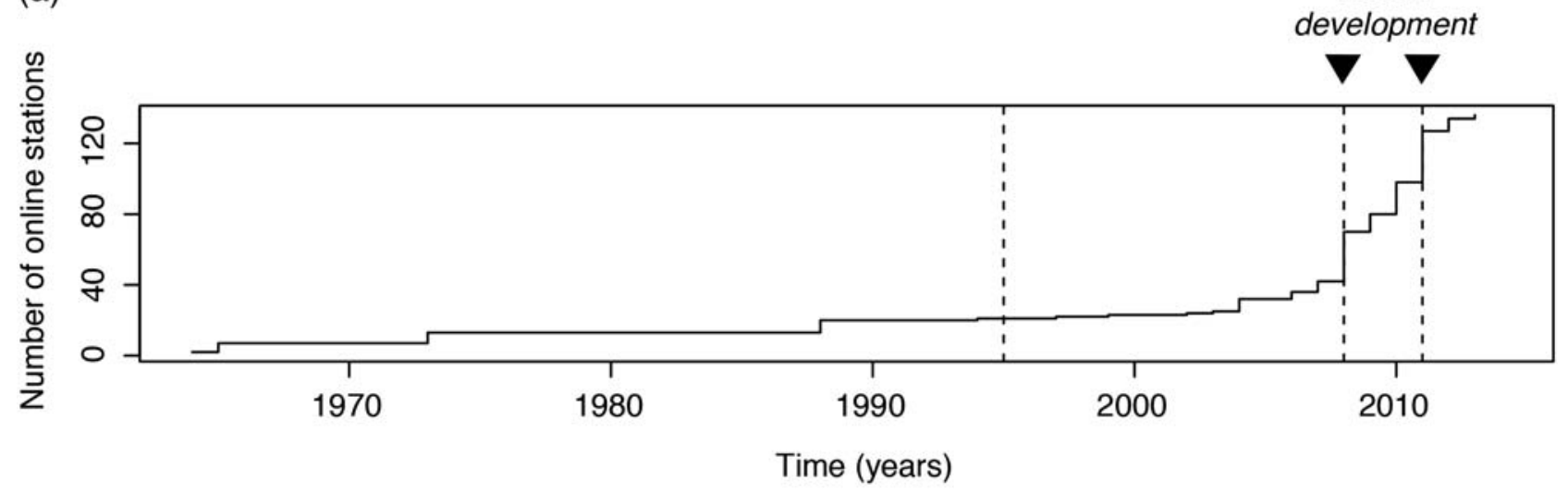

(b)

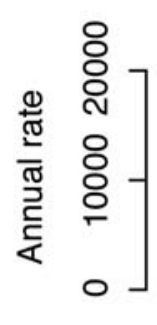

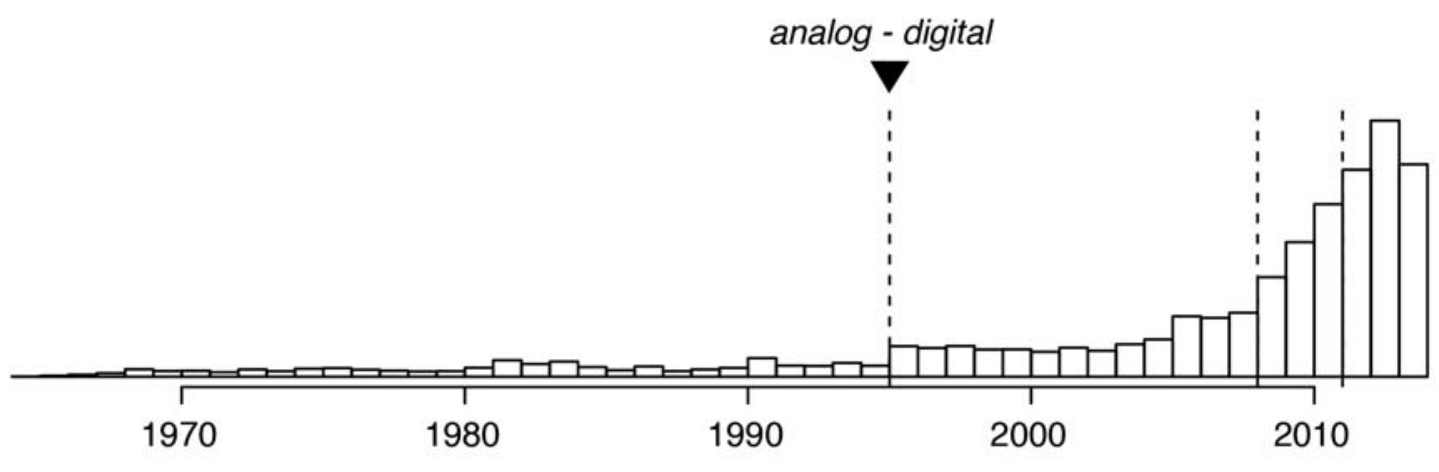

Time (years)

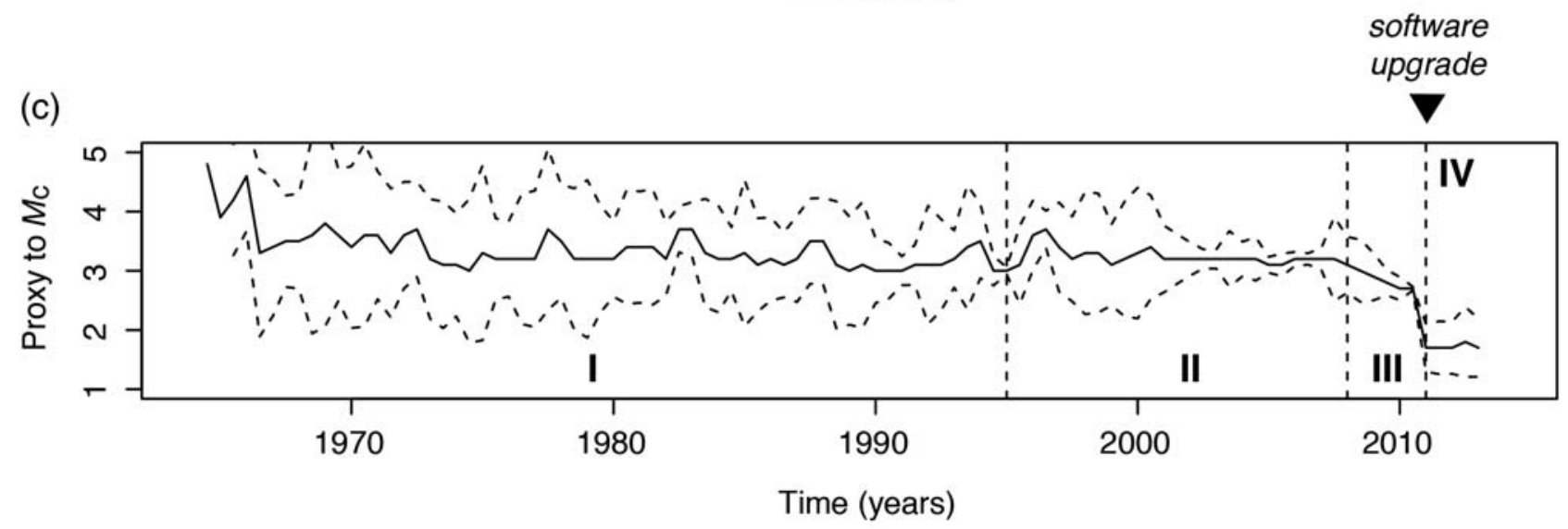

(d)

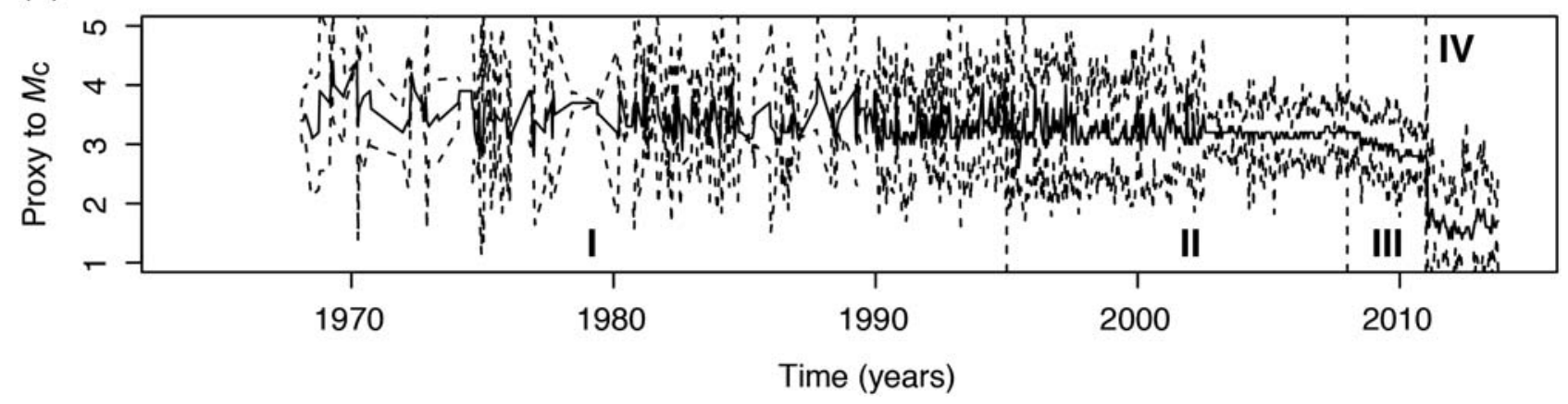

A Figure 1. 1964-2013 time series of (a) the number of online stations, (b) the annual number of events, and (c, d) a proxy to the completeness magnitude $M_{\mathrm{c}}$ defined as the regional $M_{\mathrm{c}}$ value obtained by the median-based analysis of the segment slope (MBASS) technique. The dotted curves represent the \pm 3 -sigma bounds obtained from 200 bootstrap samples. Time windows of (c) 1 year and (d) 1 month. Based on these metrics, four time intervals (I-IV) are defined. 
The BMC method is a two-step procedure. It consists of (1) a spatial resolution optimization to compute an observed $M_{\mathrm{c}}^{\text {obs }}$ map, in which the number of $M_{\mathrm{c}}$ estimates is maximized while spatial heterogeneities in $M_{\mathrm{c}}$ are minimized and (2) a Bayesian approach that combines observations and prior information. Mignan et al. (2011) defined the prior model $M_{\mathrm{c}}^{\text {pred }}=$ $f(d, k)$ with $d$ the distance to the $k$ th nearest seismic station as

$M_{\mathrm{c}}(d, k)=c_{1} d^{c_{2}}+c_{3}$,

in which parameters $c_{1}, c_{2}$, and $c_{3}$ are determined empirically. The parameter $k$ is chosen as the minimum number of stations to be triggered for initiating the location procedure in the network, usually between 3 and 5. For Taiwan, Mignan et al. (2011) found $c_{1}=5.96, c_{2}=0.0803, c_{3}=5.80$ and a standard deviation $\sigma=0.18$ for $k=4$ with $d$ in kilometers and using the local magnitude scale $M_{\mathrm{L}}$. Equation (1) has been shown to be valid in various regions, although data scattering may be high (Mignan et al., 2013), and instabilities may be observed at long distances (Vorobieva et al., 2013). So far, the Taiwanese model has been considered as the default BMC model, whereby equation (1) is best defined in terms of $\sigma$ and distance $d$ range.

The first step of the BMC method-the spatial resolution optimization procedure-consists in computing $M_{\mathrm{c}}^{\text {obs }}(x, y)$ from the FMD defined from events located in a cylindrical volume centered on cell $(x, y)$ of radius:

$r=\frac{1}{2}\left[\left(\frac{c_{1} d^{c_{2}}+\sigma}{c_{1}}\right)^{\frac{1}{c_{2}}}-\left(\frac{c_{1} d^{c_{2}}-\sigma}{c_{1}}\right)^{\frac{1}{c_{2}}}\right]$,

in which $d, c_{1}, c_{2}$, and $\sigma$ are the same parameters as in equation (1). In equation (2), $\sigma$ can be interpreted as an $M_{c}$ interval under which variations cannot be resolved. It is worth noting that Vorobieva et al. (2013) found a similar scaling law using a different approach. It follows from equation (2) that at a cell $(x, y)$ located at $d=50 \mathrm{~km}$ from the fourth nearest station, $r=14 \mathrm{~km}$; for $d=100 \mathrm{~km}, r=26 \mathrm{~km}$; and for $d=200 \mathrm{~km}$, $r=50 \mathrm{~km}$. If $r$ is smaller than half the cell diagonal distance, the FMD is computed from all earthquakes located in that cell (highest spatial resolution). The hypothesis that regions of homogeneous $M_{\mathrm{c}}$ are smaller in the dense parts of the seismic network than in the outer regions is corroborated by independent observations made by Mignan (2012a). This method avoids any arbitrary decision on parameter $r$ and any oversmoothing, which could corrupt $M_{\mathrm{c}}$ estimates (Mignan et al., 2011; Mignan and Woessner, 2012).

$M_{\mathrm{c}}$ is computed as the magnitude $m$ bin with the maximum number of events $N$ (e.g., Wiemer and Wyss, 2000), which has been shown to be valid for homogeneous datasets described by an angular FMD (Mignan, 2012a; see Concept of Elemental FMD section). In BMC, $M_{\mathrm{c}}^{\text {obs }}$ is the mean of $M_{\mathrm{c}}$ values obtained from 200 bootstrap FMD samples and $\sigma_{0}$ is the associated standard error. Event sets composed of at least four events are used because Mignan et al. (2011) demon- strated that for small sample sizes (1) uncertainty estimates based on bootstrapping are still reliable, and (2) the observed large fluctuations of $\sigma_{0}$ are an accurate reflection of how well a particular sample of magnitudes can constrain $M_{\mathrm{c}}$. Note that this is only valid for an angular FMD. For other FMD shapes, $M_{\mathrm{c}}$ estimation requires a larger set of events to reach a stable value.

The second step of BMC consists in merging prior information (equation 1) with observations, based on Bayes theorem. Following Mignan et al. (2011) and assuming a normal distribution of uncertainties, the posterior $M_{\mathrm{c}}^{\text {post }}$ is defined such that

$M_{\mathrm{c}}^{\mathrm{post}}=\frac{M_{\mathrm{c}}^{\mathrm{pred}} \sigma_{0}^{2}+M_{\mathrm{c}}^{\mathrm{obs}} \sigma^{2}}{\sigma^{2}+\sigma_{0}^{2}}$,

the average of the predicted and observed completeness magnitude, weighted according to their respective uncertainties. The posterior standard deviation $\sigma_{\text {post }}$ is given by

$\sigma_{\text {post }}=\sqrt{\frac{\sigma^{2} \sigma_{0}^{2}}{\sigma^{2}+\sigma_{0}^{2}}}$

It follows that $M_{\mathrm{c}}^{\text {obs }}$ observations have more weight in regions of low uncertainty (low $\sigma_{0}$ ) whereas prior information has more weight in region of high uncertainty. In regions where there is no observation, the prior $M_{\mathrm{c}}^{\text {pred }}$ is used. $M_{\mathrm{c}}^{\text {post }}$ is commonly referred to as the BMC estimate.

\section{Results}

We created one BMC map per time interval on a $0.1^{\circ} \times 0.1^{\circ}$ longitude-latitude grid. Although transient increases in $M_{\mathrm{c}}$ due to aftershock bursts (e.g., Ogata and Katsura, 2006; Iwata, 2008; Omi et al., 2013) are generally filtered out by the BMC method (Mignan et al., 2011), in our analysis an anomaly persisted in period I due to the 1981 Alkyonides earthquake sequence (see Transient Changes in $M_{\mathrm{c}}$ and the BMC Method section). Consequently, we generated BMC maps using a declustered version of the NOA catalog, determined by the nearest-neighbor cluster method introduced by Zaliapin et al. (2008). The $M_{\mathrm{c}}^{\text {obs }}$ spatial optimization is based on the default model, whereas $M_{\mathrm{c}}^{\text {pred }}$ corresponds to the default model calibrated to the Greek data for $k=4$. The calibration is defined by $c_{3}=c_{3 \text { (default) }}+\mu$, in which $\mu$ being the mean of the residual $M_{\mathrm{c}}^{\text {obs }}-M_{\mathrm{c}}^{\text {pred(default) }}$. Using this procedure we found $c_{3}=-5.59,-5.65,-5.62$, and -6.59 for time intervals I, II, III, and IV, respectively. Figure 2 shows the $M_{\mathrm{c}}^{\text {pred }}=f(d, 4)$ model for each one of the four periods. The one magnitude unit shift to lower $M_{\mathrm{c}}$ from period III to period IV coincides with the 2011 analysis software upgrade, which apparently improved the quality of the NOA catalog. This upgrade involved the use of the Nanometrics Atlas data processing package and routine magnitude determination by a Wood-Anderson 

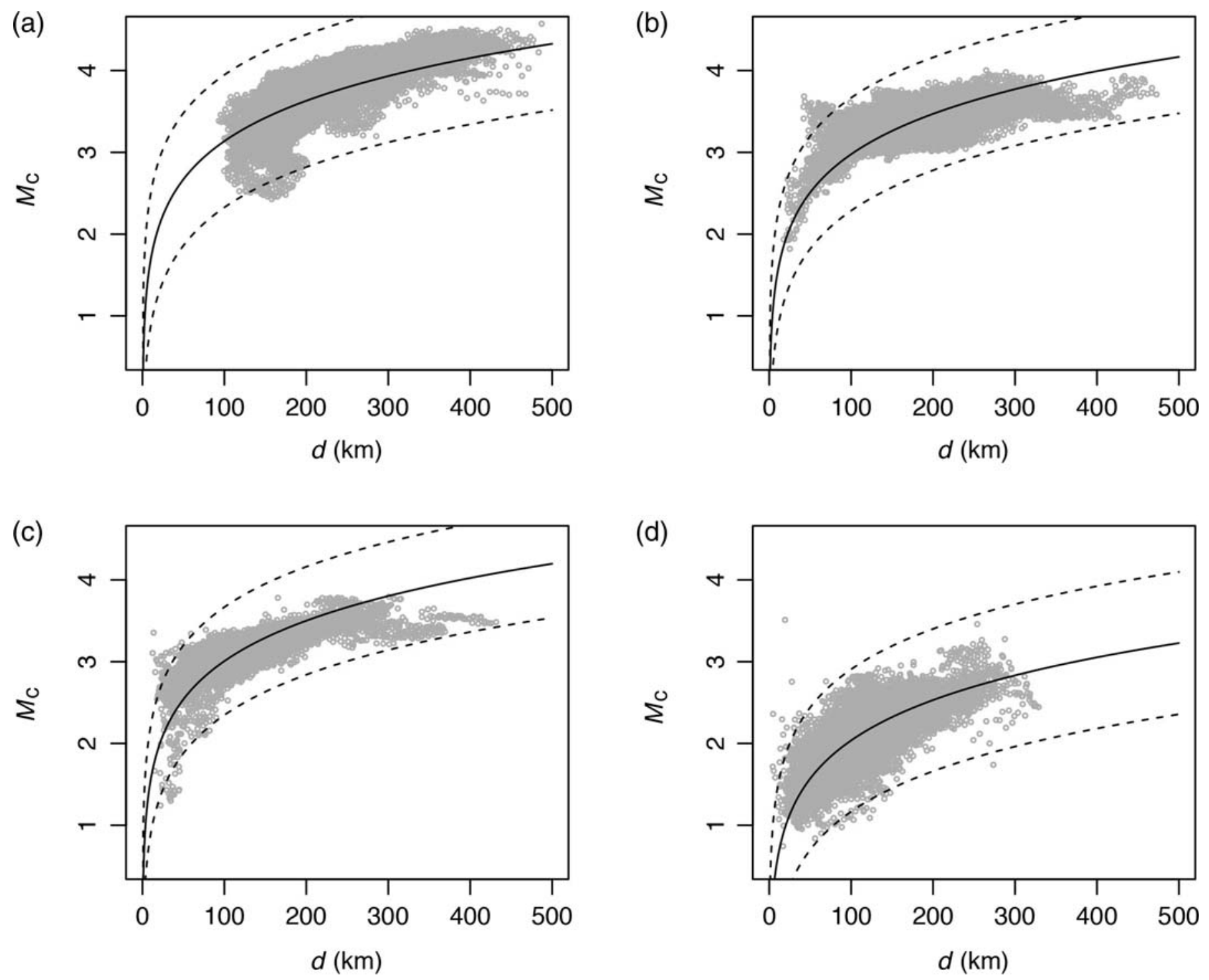

- Figure 2. $M_{\mathrm{c}}$ as a function of distance $d$ to the fourth nearest seismic station per time interval. Gray dots represent $M_{\mathrm{c}}^{\mathrm{obs}}$ and the solid curves $M_{\mathrm{c}}^{\text {pred }}$ (equation 1). The dashed curves represent $\pm 3 \sigma$. This model is used as an a priori information in the Bayesian magnitude of completeness (BMC) method. (a) Period I, (b) period II, (c) period III, and (d) period IV.

simulation for each reporting station, rather than the reporting of the Wood-Anderson magnitude of the Athens (ATH) station. Results from Figure 2 further indicate that the Greek data scattering is relatively high compared to Taiwan but lower compared with Mainland China, with $\sigma=0.27,0.23,0.22$, and 0.29 for the periods I, II, III, and IV, respectively.

Figure 3 shows maps of $M_{\mathrm{c}}^{\text {obs }}, M_{\mathrm{c}}^{\text {pred }}$, and $M_{\mathrm{c}}^{\text {post }}$ for the four periods. We found $M_{\mathrm{c}}^{\text {post }}$ (minimum; median; maximum) $=$ $(2.5 ; 3.8 ; 4.5),(1.9 ; 3.3 ; 4.1),(1.5 ; 3.1 ; 4.0)$, and $(0.5 ; 2.1$; 3.1) for periods I-IV, respectively. The dramatic change from period III to IV is clearly observed. Overall, we found a good agreement between $M_{\mathrm{c}}^{\text {obs }}$ and $M_{\mathrm{c}}^{\text {pred }}$, except for period I during which $M_{\mathrm{c}}^{\text {obs }}$ is abnormally low compared with the network spatial configuration in the region of Corinth. We suggest that this deviation represents the human factor, which is implicitly included in $\sigma$ ( $\sigma$ also includes potential location uncertainties). In this case, the anomaly is centered on the Athens prefecture, which is the highest populated area of Greece, meaning that the observatory received more alerts from the citizens and had to provide reports that were more detailed. Finally, Figure 4 shows the different uncertainty maps $\left(\sigma_{0}, \sigma\right.$, and $\left.\sigma_{\text {post }}\right)$ generated by the BMC method. We found $\sigma_{\text {post }}($ minimum; median; maximum $)=(0 ; 0.11 ; 0.27),(0 ; 0.11 ; 0.23),(0 ; 0.12 ; 0.22)$, and $(0 ; 0.14 ; 0.29)$ for periods I-IV, respectively. Period IV shows the highest uncertainties in $M_{\mathrm{c}}$, which questions the validity of the low $M_{\mathrm{c}}$ estimates compared with other periods. In any case, one can use the more conservative estimate $M_{\mathrm{c}}^{\text {post }}+3 \sigma_{\text {post }}$ as already proposed by Mignan et al. (2013). 

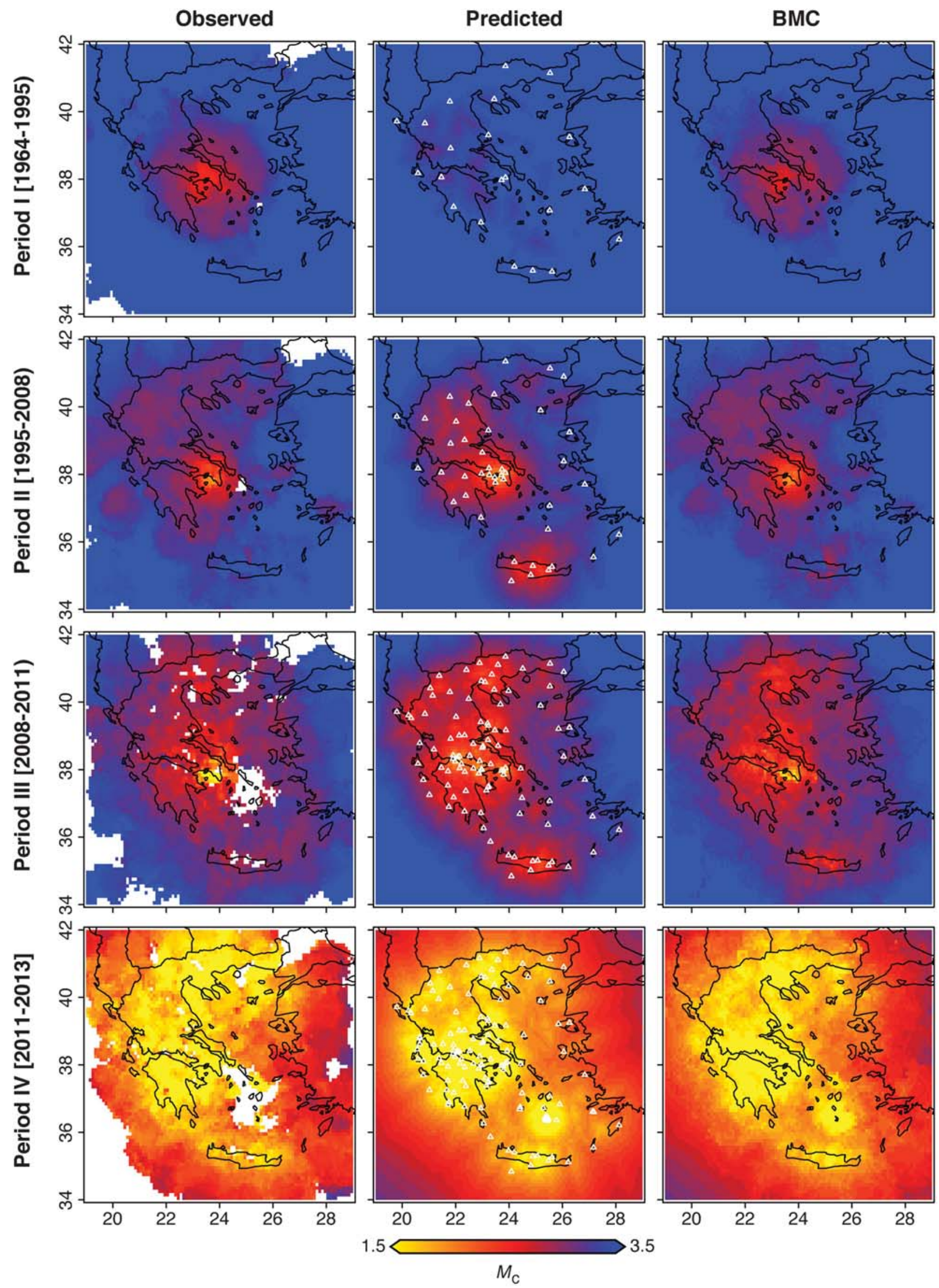

A Figure 3. $M_{\mathrm{c}}$ maps generated by the BMC method based on the declustered NOA catalog: $M_{\mathrm{c}}^{\text {obs }}$ (observed), $M_{\mathrm{c}}^{\text {pred }}$ (predicted), and $M_{\mathrm{c}}^{\text {post }}$ (BMC). 

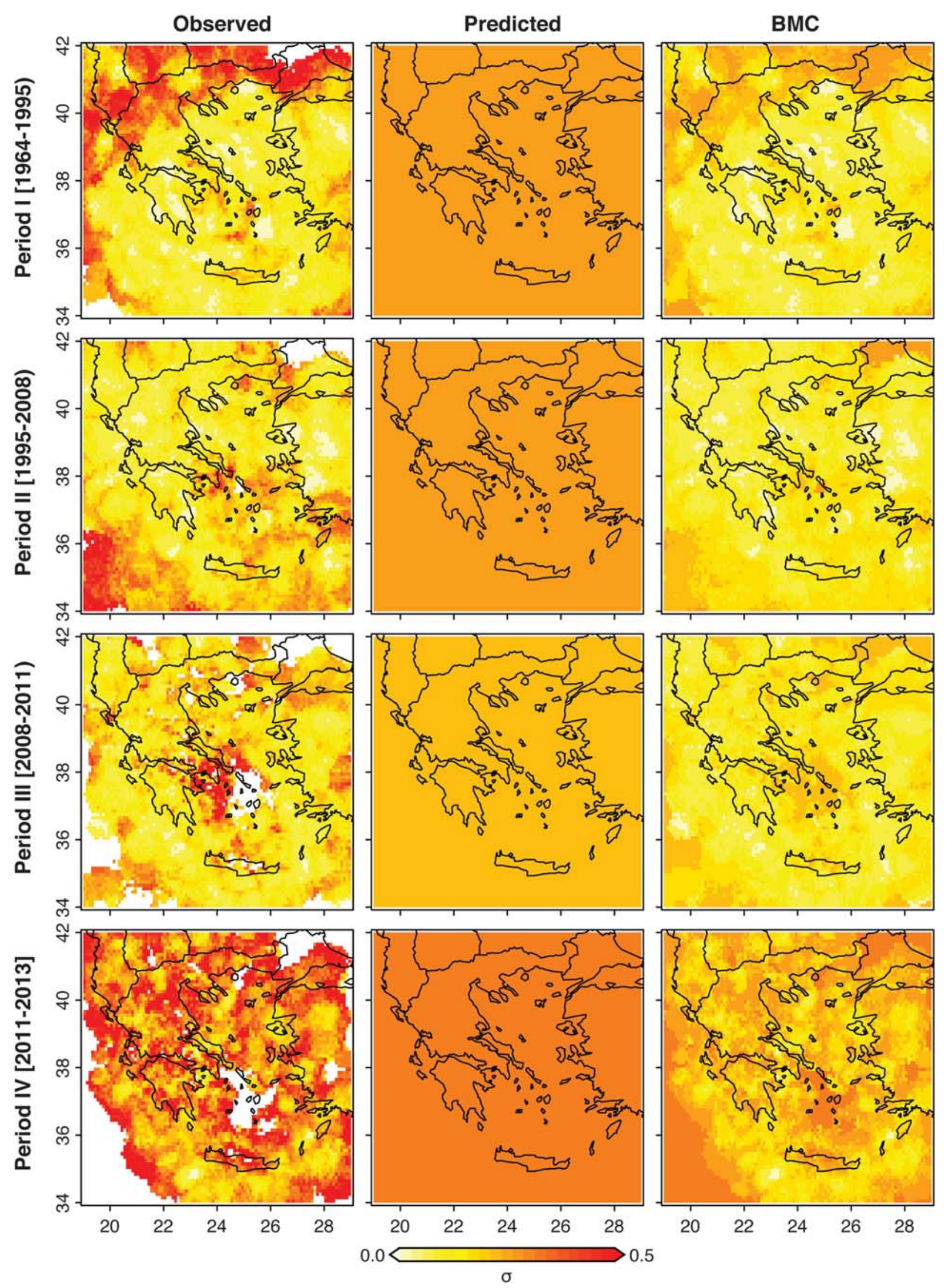

A Figure 4. $M_{\mathrm{c}}$ standard deviation maps generated by the BMC method based on the declustered NOA catalog: $\sigma_{0}$ (observed), $\sigma$ (predicted), and $\sigma_{\text {post }}(\mathrm{BMC})$. 


\section{IN-DEPTH ANALYSIS OF THE FREOUENCY- MAGNITUDE DISTRIBUTION (FMD)}

Although the analysis of the FMD is done routinely for the assessment of $M_{\mathrm{c}}$ and of the $a$ - and $b$-values of the Gutenberg-Richter law $\log _{10} N(\geq m)=a-b m$ (Gutenberg and Richter, 1944), more caution should be taken to properly assess those parameters. For instance, Mignan and Woessner (2012) showed that different well-established techniques to compute $M_{c}$ yield different results, which in turn lead to different estimates of $a$ and $b$. Mignan (2012a) also showed that the shape of the FMD is more complex than previously supposed, meaning that different FMD-based techniques may yield biased results of $M_{\mathrm{c}}, a$, and $b$ depending on the level of complexity of the data set considered. Because these parameters are the basis of numerous seismicity analyses as well as seismic-hazard assessments, we here illustrate the main issues and provide some recommendations using the Greek earthquake catalog as an example. This exercise also permits to verify the validity of the $\mathrm{BMC}$ results.

\section{Regional Detection Threshold}

Although Wiemer and Wyss (2000) already emphasized the importance of mapping $M_{\mathrm{c}}$ for a reliable estimate of the regional or bulk $M_{\mathrm{c}}$, very few studies have actually used $M_{\text {cbulk }}=\max \left(M_{\text {clocal }}\right)$. The most common approach is to compute one $M_{\mathrm{c}}$ estimate directly from the bulk FMD and consider this value as the regional detection threshold. Figure 5 shows the bulk FMDs corresponding to periods I-IV. $M_{\mathrm{c}}$ estimates from two widespread techniques-MBASS of Amorèse (a)

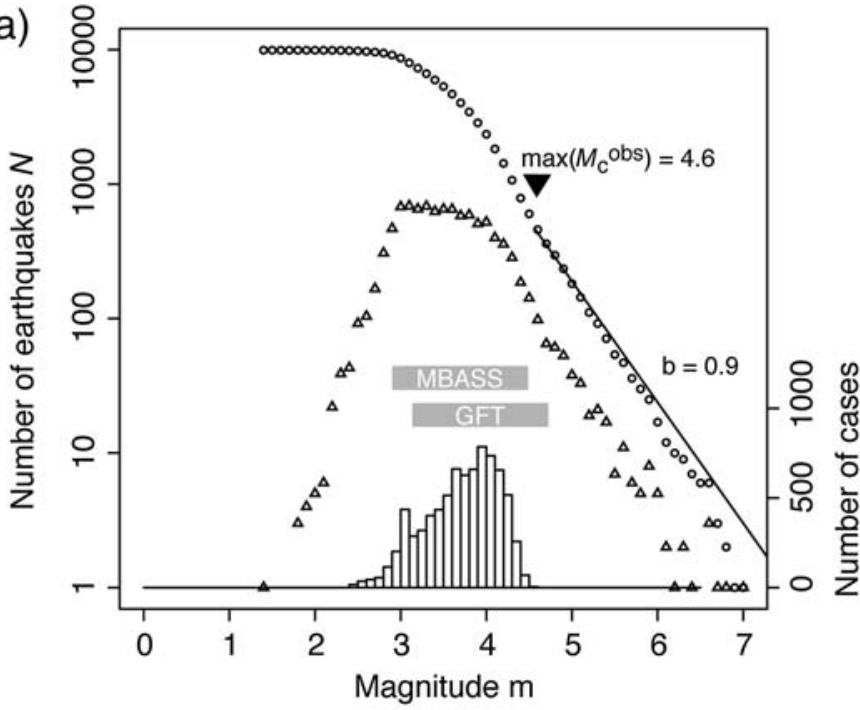

(c)

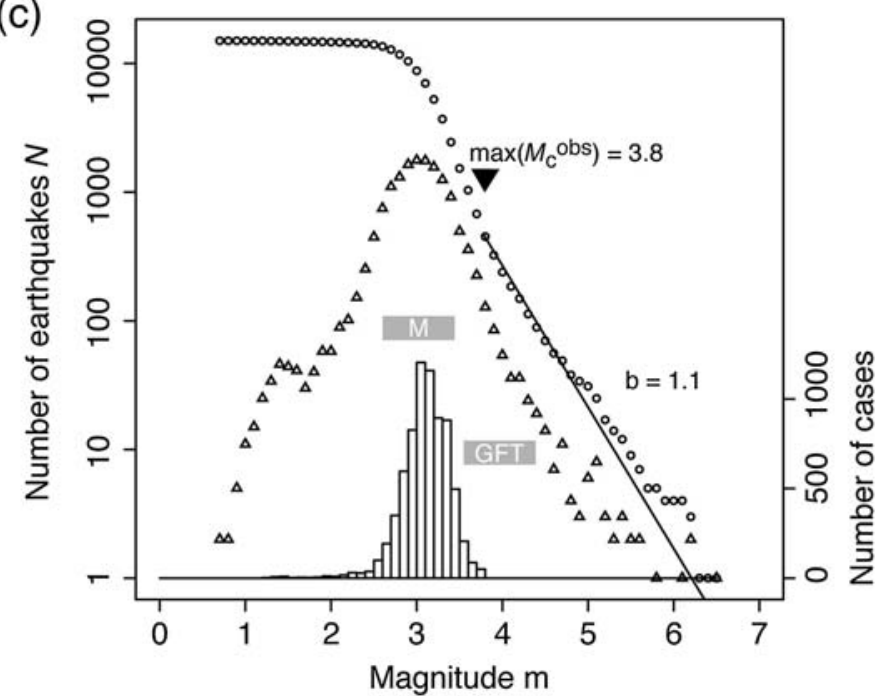

(b)

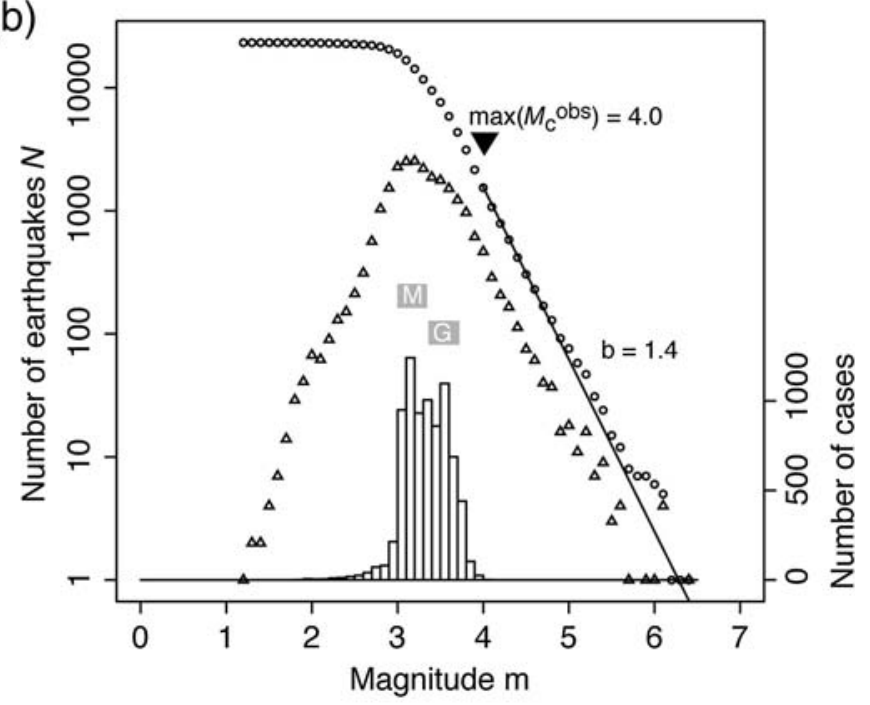

(d)

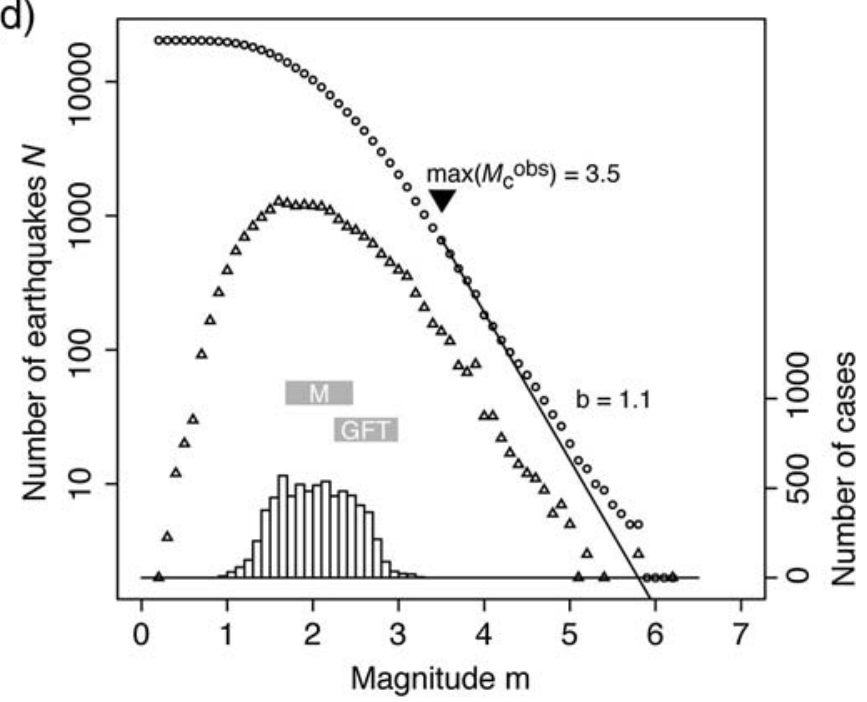

A Figure 5. Bulk FMD and $M_{\mathrm{c}}^{\text {obs }}$ distribution per time interval. The gray bars represent MBASS and GFT estimates of $M_{\mathrm{c} \text { bulk }}$ considering \pm 3 sigma over 200 bootstrap samples. The lines represent the Gutenberg-Richter law with $M_{\mathrm{c} \text { bulk }}=\max \left(M_{\mathrm{c}}^{\mathrm{obs}}\right)$. The $M_{\mathrm{c}}^{\text {obs }}$ distribution refers to the estimates shown in Figure 3. (a) Period I, (b) period II, (c) period III, and (d) period IV. 
(2007) and goodness-of-fit technique (GFT) of Wiemer and Wyss (2000) - are shown and compared to $M_{\text {cbulk }}=$ $\max \left(M_{\text {clocal }}\right)$. Here $M_{\text {clocal }}=M_{\mathrm{c}}^{\text {obs }}$ (Fig. 3) with its distribution shown on top of each bulk FMD. For MBASS and GFT, \pm 3 sigma values are given, calculated from 200 bootstrap samples. We found that MBASS and GFT estimates are in most cases lower than $\max \left(M_{\text {clocal }}\right)$. This is the reason why we considered MBASS $M_{\text {cbulk }}$ estimates as a proxy to $M_{\mathrm{c}}$ in Figures 1c and $1 \mathrm{~d}$. Although the absolute value may not always be trusted, variations over time provide an idea of the relative changes in $M_{\mathrm{c}}$. With $\max \left(M_{\text {clocal }}\right)=4.6,4.0,3.8$, and 3.5 as conservative estimates, we obtained the maximum-likelihood estimate $b=0.9,1.4,1.1$, and 0.9 for period I-IV, respectively (Aki, 1965). Following the reliability tests of Amorèse et al. (2010), we found that the $b$-values of periods I, III, and IV are undistinguishable from $b=1.0$ ( $b$ calculated using $N>400$ events) while $b=1.4$ in period II is somewhat anomalous ( $b$ calculated using $N>1500$ events).

Roumelioti et al. (2010) noted a change in the operation of the Wood-Anderson seismograph at the ATH station, from the end of 1995 to the beginning of 1996 until the abolition of the Wood-Anderson seismograph in late 2007 (our period II). They found the east-west component of the instrument started recording much larger amplitudes compared with the north-south component. This change may have a significant impact on the NOA catalog because all $M_{\mathrm{L}}$ calibrations performed in Greece until 2007 were based on $M_{\mathrm{L}}$ calculated from the maximum trace amplitudes recorded on the WoodAnderson seismograph of the ATH station. The authors concluded that this change resulted in a systematic overestimation of $M_{\mathrm{L}}$ by at least 0.1 . It remains unclear if the impact could have been greater to smaller events, which could then explain the higher $b$-value. Because this regional change matches the ATH station anomaly, it is difficult to believe that it could have a tectonic origin. It suggests that $b$-value patterns should always be interpreted with caution (e.g., Kamer and Hiemer, 2013).

\section{Concept of Elemental FMD}

Figure 5 also demonstrates the complexity of the bulk FMD shape, which can show several maxima, plateaus, and a more or less gradual curvature. This convoluted shape seems correlated to the $M_{\text {clocal }}$ distribution, in agreement with the view that any FMD could be described by the sum of elemental FMDs, an elemental FMD being defined from any space-time hypervolume of constant $M_{\mathrm{c}}$ (Mignan, 2012a). Two elemental FMD models are tested here: (1) the angular FMD model proposed by Mignan (2012a):

$\lambda\left(m \mid \kappa, \beta, M_{\mathrm{c}}\right)= \begin{cases}\exp \left[(\kappa-\beta)\left(m-M_{\mathrm{c}}\right)\right], & m<M_{\mathrm{c}}, \\ \exp \left[-\beta\left(m-M_{\mathrm{c}}\right)\right], & m \geq M_{\mathrm{c}}\end{cases}$

in which $\lambda$ is the normalized number of events (or intensity), $m$ the magnitude, $\beta=b \log (10)$, and $\kappa$ a detection parameter; and (2) the gradually curved FMD model proposed by Ogata and Katsura (1993): $\lambda(m \mid \beta, \mu, \sigma)=\exp (-\beta m) \int_{-\infty}^{m} \frac{1}{\sqrt{2 \pi} \sigma} \exp \left(-\frac{(x-\mu)^{2}}{2 \sigma^{2}}\right) d x$,

in which $\mu$ and $\sigma$ are detection parameters with $M_{\mathrm{c}}(n-\mathrm{conf})=\mu+n \sigma$ (see also Ogata and Katsura, 2006; Iwata, 2008, 2013; Omi et al., 2013). Mignan (2012a) demonstrated that the two models are inconsistent with each other with roughly $M_{\mathrm{c}}^{M}(100 \%$ events detected $)=M_{\mathrm{c}}^{\mathrm{OK}}(0$-conf; i.e., $50 \%$ events detected), which indicates the earthquake detection function is not yet clearly understood.

Although elemental FMDs are difficult to extract from earthquake catalogs due to the trade-off between the minimization of $M_{\mathrm{c}}$ heterogeneities and the maximization of the sample size, the BMC spatial optimization presented in the Method section helps optimizing this trade-off. We investigated for period III the shape of the local FMDs composed of $N \geq 100$ events for FMD model selection. We compared the angular and gradually curved FMD models using the maximum-likelihood method, as described in Mignan (2012a). Figure 6 shows two examples of local FMD and their model fits. Over the 626 event sets tested, the angular model best-fitted $42 \%$ of the local FMDs (e.g., Fig. 6a), whereas the gradually curved model fit best at $58 \%$ (e.g., Fig. 6b). Because we did not find any definitive trend in the NOA catalog, we consider each model as likely to describe an elemental FMD. It should be noted that not knowing which model is best does not hamper the use of the BMC method. Although BMC assumes that $M_{\mathrm{c}}^{\text {obs }}$ derives from the angular FMD model, using the result $M_{\mathrm{c}}^{\text {post }}+$ $3 \sigma_{\text {post }}$ permits to take into account the potential gradual curvature of a local FMD. This is illustrated in Figure 6 in which $M_{\mathrm{c}}^{\text {post }}+3 \sigma_{\text {post }}$ remains close to the maximum when the angular FMD model is preferred while it tends to $M_{\mathrm{c}}$ (3-conf) when the gradually curved model is preferred. This metric is not so sensitive to the choice of the FMD model, meaning that it should be preferred to the sole use of mean $M_{\mathrm{c}}$ estimates.

\section{Transient Changes in $\boldsymbol{M}_{\mathrm{c}}$ and the BMC Method}

The BMC method provides the long-term detection level for a fixed or at least reasonably stable seismic network. Temporary increases in $M_{\mathrm{c}}$ due to large earthquake sequences (e.g., Ogata and Katsura, 2006; Iwata, 2008; Omi et al., 2013) are supposedly not included in BMC maps. Mignan et al. (2011) verified this hypothesis in the case of the 1999 Chi-Chi, Taiwan, earthquake sequence. We, however, observed that defining $M_{\mathrm{c}}^{\text {obs }}$ as the magnitude $m$ bin with the maximum number of events $N$ failed to filter out one $M_{\mathrm{c}}$ transient in period I of the NOA catalog. Figure $7 \mathrm{a}$ shows that a high $M_{\mathrm{c}}$ anomaly is observed in the region of Corinth when computing $M_{\mathrm{c}}^{\text {obs }}$ from the original NOA catalog. This anomaly corresponds to the 1981 Alkyonides earthquake sequence (Jackson et al., 1982). One can note that this anomaly disappears once BMC is applied to the declustered version of the catalog (Fig. 3). Figure $7 \mathrm{~b}$ shows the local FMD of the anomaly area (circle in Fig. 7a). The jump in 

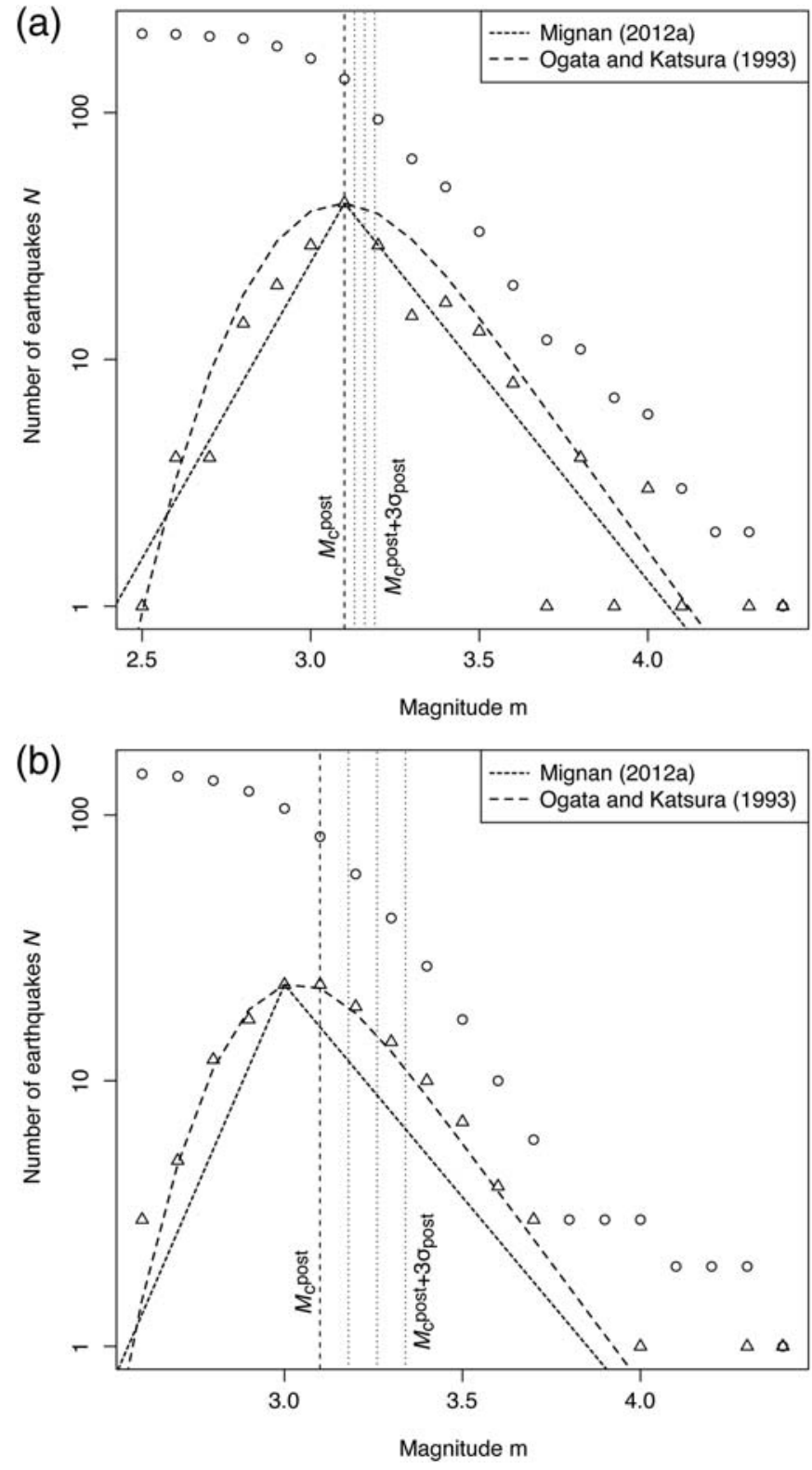

A Figure 6. Examples of local FMDs observed in period III. (a) Data fit best by the angular FMD model of Mignan (2012a); (b) data fit best by the gradually curved FMD model of Ogata and Katsura (1993). The BMC result $M_{\mathrm{c}}^{\text {post }}+3 \sigma_{\text {post }}$ permits to take into account the potential gradual curvature of a local FMD, which means that this metric is not so sensitive to the choice of the FMD model.

the number of events at $m=3.2$ due to the 1981 aftershock activity burst is such that it surpasses the maximum number of events $N(m)$ observed over all of period I in that area, which led to $M_{\mathrm{c}}=3.2$ (transient) instead of $M_{\mathrm{c}}=2.7$ (latent). It is interesting to note that the dramatic jump indicates that no aftershock was declared below the threshold $m_{\text {th }}=3.2$. A Heaviside detection function (no detection below $m_{\mathrm{th}}$, complete detection above $m_{\mathrm{th}}$ ) can be described by whether $\kappa \gg \beta$ (equation 5) or $\sigma \rightarrow 0$ (equation 6). This example again (a)
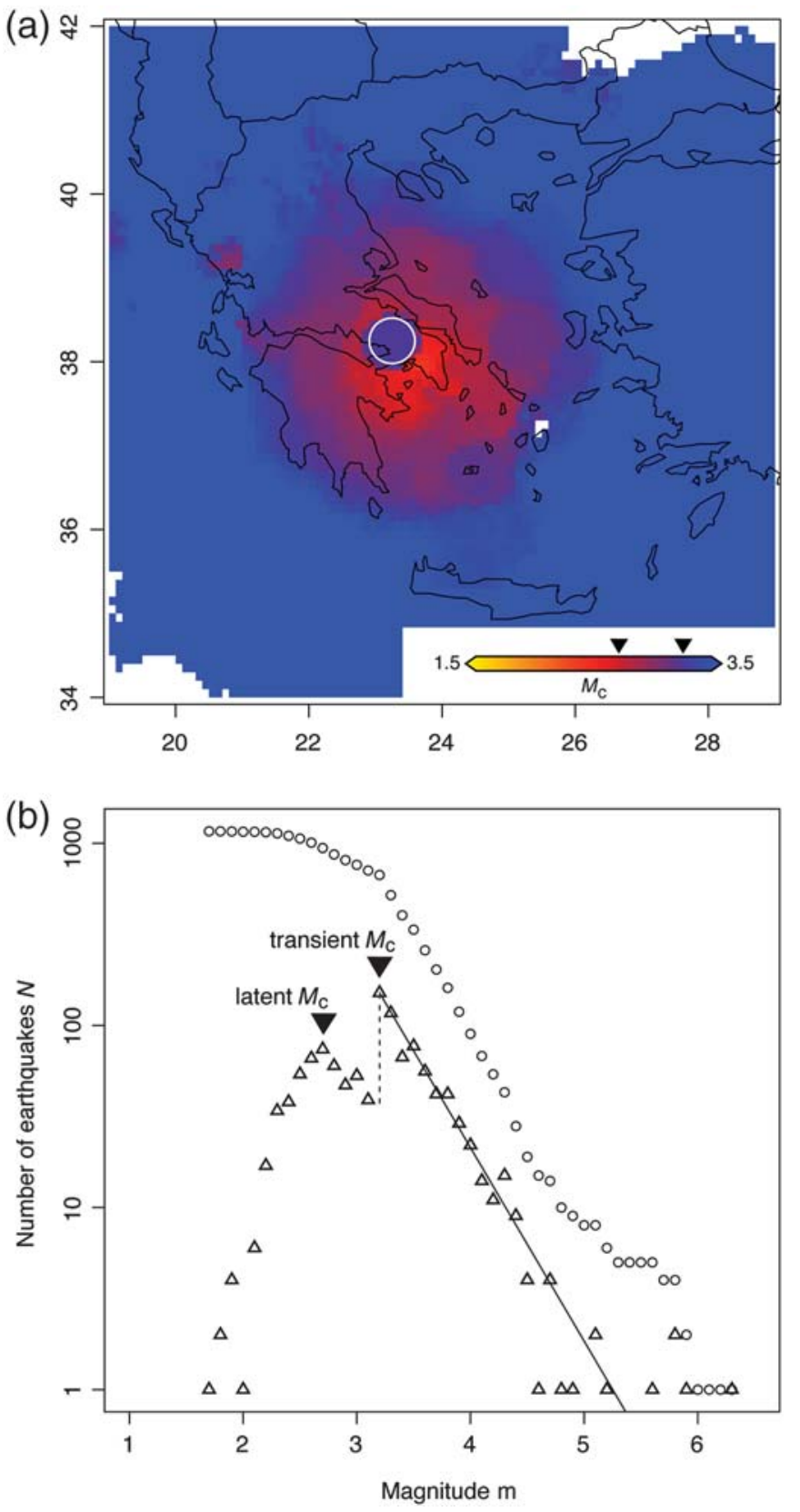

A Figure 7. Impact of the 1981 Alkyonides earthquake sequence on $M_{\mathrm{c}}$ estimates in period I. (a) BMC $M_{\mathrm{c}}^{\mathrm{obs}}$ map generated from the original NOA catalog. The white circle of $38 \mathrm{~km}$ radius highlights the contour of a high $M_{\mathrm{c}}$ anomaly resulting from the earthquake sequence. (b) FMD of the circular area. The anomaly shown on the map is explained by the dramatic increase in the number of events (i.e., aftershocks) at magnitude $m=3.2$. The line represents the Gutenberg-Richter law with slope $b=1.1$.

illustrates just how complex an FMD can be, and that only a careful inspection of the FMD shape can help the better understanding of how $M_{c}$, and therefore the $a$ - and $b$-values should be assessed. For the three other time intervals, the BMC results were almost identical with or without declustering. 


\section{CONCLUSIONS}

Maps of $M_{\mathrm{c}}$ and $M_{\mathrm{c}}$ standard deviations were produced based on the BMC method for the NOA earthquake catalog for the four time intervals: 1964-1994 (I), 1995-2007 (II), 20082010 (III), and 2011-present (IV). $M_{\mathrm{c}}$ (minimum; median; maximum) was shown to evolve from values of $(2.5 ; 3.8$; $4.5)$ to $(0.5 ; 2.1 ; 3.1)$ from period I to IV. These results are subject to uncertainty with a standard deviation (minimum; median; maximum) of $(0 ; 0.14 ; 0.29)$. Our results showed the dramatic improvement in the seismic network performance since 2011, which is apparently linked to a software upgrade. This change should enable better microseismicity analyses in the foreseeing future, which promote a better understanding of complex fault structures and physical processes (e.g., Pacchiani and Lyon-Caen, 2010) and improvement of earthquake forecasting skills (Mignan, 2012b; Papadopoulos et al., 2006).

Based on the different results obtained from the BMC method and the FMD shape investigation, we provide the following general recommendations for $M_{\mathrm{c}}$ estimation:

1. Use $M_{\text {cbulk }}=\max \left(M_{\text {clocal }}\right)$ based on a mapping method (e.g., BMC) as a conservative estimate of the regional detection threshold.

2. Use $M_{\mathrm{c}}^{\text {post }}+3 \sigma_{\text {post }}$ based on the BMC method for local estimates, in order to take into account the different possible shapes of local FMD.

3. Systematically investigate the shape of the FMD (regional and/or local) as human errors and transient processes may always affect the evaluation of $M_{\mathrm{c}}$ and of the $a$ and $b$-values. $\mathbb{Z}$

\section{ACKNOWLEDGMENTS}

We thank Editor Zhigang Peng as well as Antonino D'Alessandro and an anonymous reviewer for their valuable comments. We are also grateful to the National Observatory of Athens (NOA) for making their catalog publicly available.

\section{REFERENCES}

Aki, K. (1965). Maximum likelihood estimate of $\mathrm{b}$ in the formula $\log N=a-b M$ and its confidence limits, Bull. Earthq. Res. Inst. Univ. Tokyo 43, 237-239.

Amorèse, D. (2007). Applying a change-point detection method on frequency-magnitude distributions, Bull. Seismol. Soc. Am. 97, 1742-1749, doi: 10.1785/0120060181.

Amorèse, D., J.-R. Grasso, and P. A. Rydelek (2010). On varying $b$-values with depth: Results from computer-intensive tests for Southern California, Geophys. J. Int. 180, 347-360, doi: 10.1111/j.1365246X.2009.04414.x.

Båth, M. (1983). The seismology of Greece, Tectonophysics 98, 165-208.

Chouliaras, G., and G. N. Stavrakakis (1997). Seismic source parameters from a new dial-up seismological network in Greece, Pure Appl. Geophys. 150, 91-111.

Chouliaras, G., N. S. Melis, G. Drakatos, and K. Makropoulos (2013). Operational network improvements and increased reporting in the NOA (Greece) seismicity catalog, Adv. Geosci. 36, 7-9, doi: 10.5194/adgeo-36-7-2013.
D’Alessandro, A., D. Papanastassiou, and I. Baskoutas (2011). Hellenic Unified Seismological Network: An evaluation of its performance through SNES method, Geophys. J. Int. 185, 1417-1430.

Deshcherevskii, A. V., and A. Ya. Sidorin (2012). Changes in representativity of the earthquake catalogue for Greece in time and space, Seism. Instrum. 48, 292-302.

Gutenberg, B., and C. F. Richter (1944). Frequency of earthquakes in California, Bull. Seismol. Soc. Am. 34, 184-188.

Iwata, T. (2008). Low detection capability of global earthquakes after the occurrence of large earthquakes: Investigation of the Harvard CMT catalogue, Geophys. J. Int. 174, 849-856, doi: 10.1111/ j.1365-246X.2008.03864.x.

Iwata, T. (2013). Estimation of completeness magnitude considering daily variation in earthquake detection capability, Geophys. J. Int. 194, 1909-1919, doi: 10.1093/gji/ggt208.

Jackson, J. A., J. Gagnepain, G. Houseman, G. C. P. King, P. Papadimitriou, C. Soufleris, and J. Virieux (1982). Seismicity, normal faulting, and the geomorphological development of the Gulf of Corinth (Greece): The Corinth earthquakes of February and March 1981, Earth Planet. Sci. Lett. 57, 377-397.

Kamer, Y., and S. Hiemer (2013). Comment on "Analysis of the b-values before and after the 23 October $2011 M_{\mathrm{w}} 7.2$ Van-Ercis, Turkey, earthquake", Tectonophysics 608, 1448-1451, doi: 10.1016/ j.tecto.2013.07.040.

Kraft, T., A. Mignan, and D. Giardini (2013). Optimization of a largescale microseismic monitoring network in northern Switzerland, Geophys. J. Int. 195, 474-490, doi: 10.1093/gji/ggt225.

Mignan, A. (2012a). Functional shape of the earthquake frequencymagnitude distribution and completeness magnitude, J. Geophys. Res. 117, no. B08302, doi: 10.1029/2012JB009347.

Mignan, A. (2012b). Seismicity precursors to large earthquakes unified in a stress accumulation framework, Geophys. Res. Lett. 39, L21308, doi: $10.1029 / 2012$ GL053946.

Mignan, A., and J. Woessner (2012). Estimating the magnitude of completeness for earthquake catalogs, in Community Online Resource for Statistical Seismicity Analysis, doi: 10.5078/corssa-00180805, available at http://www.corssa.org (last accessed March 2014).

Mignan, A., C. Jiang, J. D. Zechar, S. Wiemer, Z. Wu, and Z. Huang (2013). Completeness of the Mainland China earthquake catalog and implications for the setup of the China Earthquake Forecast Testing Center, Bull. Seismol. Soc. Am. 103, 845-859, doi: 10.1785/0120120052.

Mignan, A., M. J. Werner, S. Wiemer, C.-C. Chen, and Y.-M. Wu (2011). Bayesian estimation of the spatially varying completeness magnitude of earthquake catalogs, Bull. Seismol. Soc. Am. 101, 1371-1385, doi: 10.1785/0120100223.

Ogata, Y., and K. Katsura (1993). Analysis of temporal and spatial heterogeneity of magnitude frequency distribution inferred from earthquake catalogues, Geophys. J. Int. 113, 727-738.

Ogata, Y., and K. Katsura (2006). Immediate and updated forecasting of aftershock hazard, Geophys. Res. Lett. 33, L10305, doi: 10.1029/ 2006GL025888.

Omi, T., Y. Ogata, Y. Hirata, and K. Aihara (2013). Forecasting large aftershocks within one day after the main shock, Scientif. Rept. 3, 2218, doi: 10.1038/srep02218.

Pacchiani, F., and H. Lyon-Caen (2010). Geometry and spatio-temporal evolution of the 2001 Agios Ioanis earthquake swarm (Corinth Rift, Greece), Geophys. J. Int. 180, 59-72, doi: 10.1111/j.1365246X.2009.04409.x.

Papadopoulos, G. A., I. Latoussakis, E. Daskalaki, G. Diakogianni, A. Fokaefs, M. Kolligri, K. Liadopoulou, K. Orfanogionnaki, and A. Pirentis (2006). The East Aegean Sea strong earthquake sequence of October-November 2005: Lessons learned for earthquake prediction from foreshocks, Nat. Hazards Earth Sci. 6, 895-901.

Papanastassiou, D. (2010). Earthquake detection-Location capability of the Hellenic Unified Seismological Network (HUSN) operating by 
the Institute of Geodynamics, Natl. Observ. Athens, Hellenic J. Geosci. 45, 209-216.

Papanastassiou, D., J. Latoussakis, and G. Stavrakakis (2001). A revised catalogue of earthquakes in the broader area of Greece for the period 1950-2000, Proc. 9th Int. Congress Geol. Soc. Greece, Athens, Greece, 26-28 September 2001, Vol. XXXIV/4, 1563-1566.

Roumelioti, Z., A. Kiratzi, and C. Benetatos (2010). The instability of the $M_{\mathrm{w}}$ and $M_{\mathrm{L}}$ comparison for earthquakes in Greece for the period 1969 to 2007, J. Seismol. 14, 309-337, doi: 10.1007/ s10950-009-9167-x.

Vorobieva, I., C. Narteau, P. Shebalin, F. Beauducel, A. Nercessian, V. Clouard, and M.-P. Bouin (2013). Multiscale mapping of completeness magnitude of earthquake catalogs, Bull. Seismol. Soc. Am. 103, 2188-2202, doi: 10.1785/0120120132.

Wiemer, S., and M. Wyss (2000). Minimum magnitude of completeness in earthquake catalogs: Examples from Alaska, the Western United States, and Japan, Bull. Seismol. Soc. Am. 90, 859-869.

Zaliapin, I., A. Gabrielov, V. Keilis-Borok, and H. Wong (2008). Clustering analysis of seismicity and aftershock identification,
Phys. Rev. Lett. 101, 018501, doi: 10.1103/PhysRevLett.101.018501.

Arnaud Mignan

Swiss Seismological Service ETH Zurich, NO H66 Sonneggstrasse 5 8092 Zurich, Switzerland arnaud.mignan@sed.ethz.ch

Gerasimos Chouliaras Institute of Geodynamics National Observatory of Athens P.O. Box 20048 11810 Athens, Greece g.choul@noa.gr 